\title{
Effectiveness and safety of sofosbuvir-based regimens plus an NS5A inhibitor for patients with HCV genotype 3 infection and cirrhosis: results of a multicenter real-life cohort
}

S. Alonso ${ }^{1}$, M. Riveiro-Barciela ${ }^{2,3}$, I. Fernandez $^{4}$, D. Rincón $^{5,3}$, Y. Real $^{6}$, S. Llerena ${ }^{7}, \mathrm{~F}$. $\mathrm{Gea}^{8}$, A. Olveira ${ }^{9,3}$, C. Fernandez-Carrillo ${ }^{10,3}$, B. Polo ${ }^{11}$, J. A. Carrión ${ }^{12}$, A. Gómez ${ }^{13}$, M. J. Devesa ${ }^{14}$, C. Baliellas ${ }^{15}$, Á. Castro ${ }^{16}$, J. Ampuero ${ }^{17,3}$, R. Granados ${ }^{18}$, J. M.

Pascasio $^{19,3}$, A. Rubín ${ }^{20}$, J. Salmeron ${ }^{21}$, E. Badia ${ }^{22}$, J. M. M. Planas ${ }^{23}$, S. Lens ${ }^{24,3}$, J. Turnes $^{25}$, J. L. Montero ${ }^{26}$, M. Buti ${ }^{2,3}$, R. Esteban ${ }^{2,3}$, C. M. Fernández-Rodríguez ${ }^{1}$

${ }^{1}$ Gastroenterology, Hospital Universitario Fundación Alcorcón, Madrid, Spain

${ }^{2}$ Liver Unit, Internal Medicine Department, Vall d'Hebron Hospital, Barcelona, Spain

${ }^{3}$ Centro de Investigación Biomédica en Red de Enfermedades Hepáticas y Digestivas (CIBERehd), Instituto de Salud Carlos III, Madrid, Spain

${ }^{4}$ Digestive Diseases Service, Hospital 12 Octubre, Madrid

${ }^{5}$ Hospital General Universitario Gregorio Marañón, Madrid, Spain

${ }^{6}$ Hospital Universitario La Princesa, Madrid, Spain

${ }^{7}$ Gastroenterology and Hepatology Unit, Hospital Universitario Marqués de Valdecilla, IDIVAL, Santander, Spain

${ }^{8}$ Gastroenterology and Hepatology Department, Hospital Universitario Ramón y Cajal, IRYCIS, Madrid, Spain

${ }^{9}$ Hospital Universitario La Paz, Madrid, Spain

${ }^{10}$ Liver Unit, Hospital Universitario Puerta de Hierro-Majadahonda, IDIPHIM, CIBERehd, Majadahonda, Madrid, Spain

${ }^{11}$ Hospital Universitario Fundación Jiménez Díaz, Madrid, Spain

${ }^{12}$ Liver Section, Gastroenterology Department, Hospital del Mar, Universitat Autònoma de Barcelona, IMIM (Hospital del Mar Medical Research Institute), Barcelona,

Spain

${ }^{13}$ Hospital Universitario Donostia, Donostia, Spain

${ }^{14}$ Hospital Universitario Clínico San Carlos, Madrid, Spain

${ }^{15}$ Hospital Universitari de Bellvitge, IDIBELL, Barcelona, Spain

${ }^{16}$ Hospital Universitario de A Coruña, A Coruña, Spain

${ }^{17}$ Hospital Virgen de Valme, and Institute of Biomedicine of Seville, Spain

${ }^{18}$ H. U. de Gran Canaria Dr. Negrín, Gran Canaria, Spain

${ }^{19}$ H. U. Virgen del Rocío, Seville, Spain

${ }^{20}$ Hepatology Unit, Digestive Medicine Service, Hospital Universitari i Politècnic La Fe, Valencia, Spain

${ }^{21}$ H. U. San Cecilio, Granada, Spain

${ }^{22}$ Hospital Universitario de Burgos, Burgos, Spain

${ }^{23}$ C. H. U. de Albacete, Albacete, Spain

${ }^{24}$ Liver Unit, Hospital Clínic, Instituto de Investigaciones Biomédicas August Pi i Sunyer (IDIBAPS), Barcelona, Spain

${ }^{25}$ Department of Gastroenterology, Complejo Hospitalario Universitario de Pontevedra and IISGS, Pontevedra, Spain

${ }^{26}$ Hospital Reina Sofia, Córdoba, Spain 


\section{Summary}

Patients with HCV genotype 3 (GT3) infection and cirrhosis are currently the most difficult to cure. We report our experience with sofosbuvir+daclatasvir $(\mathrm{SOF}+\mathrm{DCV})$ or sofosbuvir/ledipasvir (SOF/LDV), with or without ribavirin (RBV) in clinical practice in this population. This was a multicenter observational study including cirrhotic patients infected by HCV GT3, treated with sofosbuvir plus an NS5A inhibitor (May 2014-October 2015). In total, 208 patients were included: 98 (47\%) treatment-experienced, 42 (20\%) decompensated and $55(27 \%)$ MELD score $>10$. In $131(63 \%)$, treatment was SOF+DCV and in $77(37 \%)$, SOF/LDV. Overall, $86 \%$ received RBV. RBV addition and extension to 24 weeks was higher in the SOF/LDV group (95\% vs $80 \%, \mathrm{P}=.002$ and $83 \%$ vs $72 \%, \mathrm{P}=.044$, respectively). A higher percentage of decompensated patients were treated with DCV than LDV (25\% vs $12 \%$, $\mathrm{P}=.013)$. Overall, SVR12 was $93.8 \%$ (195/208): $94 \%$ with SOF+DCV and 93.5\% with SOF/LDV. SVR12 was achieved in $90.5 \%$ of decompensated patients. Eleven treatment failures: 10 relapses and one breakthrough. RBV addition did not improve SVR (RR: 1.08; $\mathrm{P}=.919)$. The single factor associated with failure to achieve SVR was platelet count $<75 \times 10 \mathrm{E} 9 / \mathrm{mL}$ (RR: $3.50, \mathrm{P}=.019$ ). In patients with MELD <10, type of NS5A inhibitor did not impact on SVR12 (94\% vs 97\%; adjusted RR: 0.49). Thirteen patients $(6.3 \%)$ had serious adverse events, including three deaths $(1.4 \%)$ and one therapy discontinuation $(0.5 \%)$, higher in decompensated patients $(16.7 \%$ vs $3.6 \%$, $\mathrm{P}<.006)$. In patients with GT3 infection and cirrhosis, SVR12 rates were high with both SOF+DCV and SOF/LDV, with few serious adverse events.

Keywords

Cirrhosis, daclatasvir, genotype 3, hepatitis C, ledipasvir, observational study, real-world cohort, sofosbuvir, SVR12

\section{INTRODUCTION}

Patients with hepatitis $\mathrm{C}$ virus (HCV) genotype 3 (GT3) infection are at a higher risk of progression to liver disease and development of hepatocellular carcinoma than patients with other HCV genotypes. ${ }^{1-3}$ Hence, this population should be prioritized to receive effective antiviral therapy. Direct-acting antiviral (DAA) agents have significantly improved the efficacy and tolerability of HCV treatment. However, as compared to other genotypes, the efficacy of DAA combinations is lower in patients with GT3 infection and liver cirrhosis.

Sofosbuvir $^{4}$ (SOF), a pangenotypic NS5B inhibitor, and daclatasvir ${ }^{5}$ (DCV), an NS5A inhibitor, are active against GT3 infection, whereas ledipasvir ${ }^{6}$ (LDV), another NS5A inhibitor, has shown lower in vitro activity against GT3. ${ }^{7}$ There are few studies evaluating these regimes in GT3-associated cirrhosis in real practice settings.

The ALLY-3 clinical trial assessed the efficacy of the SOF+DCV combination for 12 weeks in GT3 patients with and without cirrhosis. The sustained virological response rate after 12 weeks of therapy (SVR12) in cirrhotic patients was 63\% (58\% in treatment-naïve [TN] and 69\% in treatment-experienced [TE] patients). ${ }^{8}$ A later study, ALLY-3+, was designed to evaluate the effect of adding ribavirin (RBV) and extending treatment duration to 16 weeks in patients with advanced fibrosis. SVR12 rates increased to $88 \%$ with SOF+DCV and RBV for 12 weeks, and to $86 \%$ with extension of therapy to 16 weeks. ${ }^{9}$

In a trial including 101 patients with GT3 infection, the fixed-dose combination of SOF/LDV without RBV achieved an SVR12 rate of $64 \%$ in naïve patients. Addition of RBV improved these results in noncirrhotic patients $(100 \% \mathrm{TN}$ vs $82 \% \mathrm{TE})$ and in patients with cirrhosis (100\% TN vs $73 \% \mathrm{TE})$, although only 15 patients were included. ${ }^{10}$

In clinical practice, a larger amount of information is available for the SOF+DCV combination than for SOF/LDV. The French multicenter compassionate use programme in cirrhotic GT3 patients receiving SOF+DCV with or without RBV for 24 weeks reported SVR12 rates of $86 \%$ without RBV and $81 \%$ with RBV. Treatment of decompensated cirrhotic patients resulted in even lower SVR rates (approximately $70 \%) .{ }^{11}$ The British expanded access programme focusing on decompensated GT3 patients reported SVR 
rates of 59\% in 61 patients who received SOF/LDV and RBV for 12 weeks compared with $70 \%$ in 114 patients who received SOF+DCV and RBV for 12 weeks. ${ }^{12}$ Data are limited regarding extending SOF/LDV and RBV therapy to 24 weeks in patients with cirrhosis. The aim of this study was to evaluate the effectiveness and safety of 12- and 24-week regimens of SOF plus an NS5A inhibitor (DCV or LDV) with or without RBV in a cohort of HCV GT3-infected patients with liver cirrhosis treated in a real-life multicenter setting.

\section{MATERIALS AND METHODS}

\subsection{Study design and patient selection}

This is a multicenter observational study in a cohort of HCV GT3-infected patients with both compensated and decompensated cirrhosis who received oral DAAs in routine clinical practice. Data were collected through a National Registry (HEPA-C) under the auspices of the Spanish Association for the Study of the Liver (AEEH) and the Networked Biomedical Research Centre for the Study of the Liver and Digestive Diseases in Spain (CIBERehd and the Community of Madrid regional registry). Twentyfive Spanish centres provided data. The information included was from patients seen between May 2014 and October 2015 who had reached week 12 after completion of treatment. For the present analysis, we included data from all cirrhotic patients infected by HCV GT3 who received treatment with SOF plus an NS5A inhibitor (DCV or LDV) with or without RBV and were followed up for at least 12 weeks after therapy discontinuation. The decision to treat and the choice of treatment were entirely at the discretion of the attending physician. The therapeutic regimen was a fixed-dose combination of SOF $400 \mathrm{mg} / \mathrm{LDV}$ $90 \mathrm{mg}$ daily or SOF $400 \mathrm{mg}+\mathrm{DCV} 60 \mathrm{mg}$. The two regimens were administered for either 12 or 24 weeks and included or not a weight-based RBV dose, depending on each patient's individual clinical characteristics. The study was conducted in accordance with Good Clinical Practice guidelines and was approved by the Research Ethics Committee of Hospital Universitario Fundación Alcorcón (Madrid, Spain). All data were anonymized, and patients gave their consent to contribute to the registries.

Eligible patients were treatment-naïve or interferon-based treatment-experienced adults ( $>18$ years old) with chronic HCV GT3 infection and cirrhosis confirmed by liver biopsy, liver stiffness measurement (Fibroscan) $\geq 14.6 \mathrm{kPa}$, or $10-14.6 \mathrm{kPa}$, and the presence of known clinical signs of portal hypertension (e.g., oesophageal varices) or clinical decompensation (ascites or hepatic encephalopathy). Decompensated patients were defined as those classified as Child-Pugh-Turcotte (CPT) class B or C at the beginning of therapy, with no upper limit of the CPT score for inclusion. The key exclusion criteria were infection with genotypes other than GT3, human immunodeficiency virus or hepatitis B virus coinfection, no evidence of cirrhosis, prior therapy with direct antiviral agents and refusal to consent to the data registry.

\subsection{Efficacy and safety monitoring}

Viral load was determined using the COBAS TaqMan HCV assay, version 2.0 (Roche Molecular Systems, Inc., Indianapolis, IN), with a lower limit of quantification of 15-43 IU/mL and a lower limit of detection of $10 \mathrm{IU} / \mathrm{mL}$. HCV RNA levels were measured at baseline and at weeks 4, 12 and 24, and at 12 weeks after completion of treatment.

The primary endpoint was sustained virological response, defined as the percentage of patients with undetectable HCV RNA at week 12 after completion of treatment in each therapy regimen.

The secondary endpoints included evaluation of covariates such as liver function, portal hypertension, previous therapy, type of previous therapies, treatment regimen associated with SVR12 and discontinuation of treatment, and therapy-related serious adverse events. 
Virological relapse was established when HCV RNA was detected during follow-up in a patient who had undetectable HCV RNA at the end of the treatment. Virological breakthrough was defined as a confirmed HCV RNA increase of at least $1 \log \mathrm{IU} / \mathrm{mL}$ from the nadir value or a confirmed quantifiable HCV RNA level in a patient with undetectable HCV RNA during treatment.

Adverse events were recorded throughout the treatment period and the 12 weeks of follow-up. Serious adverse events (specifically, any hospital admission or decompensation event), death, occurrence of hepatocellular carcinoma (HCC), liver transplant requirement or treatment discontinuation related to adverse events were included in the safety analysis.

\subsection{Statistical analysis}

Covariates classically associated with virological response such as age, sex, liver stiffness, platelet count, HCV RNA and previous HCV treatment were collected for analysis. Patients with liver stiffness $>20 \mathrm{kPa}$ were considered at risk of having clinically significant portal hypertension. ${ }^{13,14}$

Results were analysed after stratifying patients by liver function according to a model for end-stage liver disease (MELD) score $>10$, Child-Pugh-Turcotte (CPT) score $\geq 7$ or albumin concentration $<3.5 \mathrm{~g} / \mathrm{dL}$ and to covariates related to HCV treatment, that is, duration of the regimen (12 or 24 weeks) and addition of RBV. Data are expressed as the mean \pm standard deviation (SD) for normally distributed quantitative variables and as the median and interquartile range for variables with a non-normal distribution. Qualitative data are expressed as the count and percentage. Efficacy endpoints, expressed as the response rate and exact binomial 95\% confidence interval, were calculated using the Clopper-Pearson method (exact method) and included patients who maintained therapy for at least 4 weeks. Univariate analysis was performed to detect differences between the treatment groups and identify predictors of treatment failure. Quantitative variables were compared with the Student $t$ test or Mann-Whitney $U$ test, according to the distribution of data. Qualitative variables were compared with the chi-square or Fisher's exact test. Risk ratios (RR) and confidence intervals were estimated using a modified Poisson model with robust error variances. ${ }^{15}$ Confounding and/or interaction effects due to liver function were analysed by subgroup analysis and multivariate models. The Mantel-Haenszel test for homogeneity was applied, and modified Poisson multivariate models were adjusted according to the type of treatment, the effectmodifying variables, and the interaction coefficient. A significant interaction indicated that the effect of treatment on SVR12 depended on the value of the other variable. The adjusted RR and risk difference were estimated using these models in each subgroup. Results were considered statistically significant at $P$ values of $<.05$. The statistical analysis was carried out using STATA 13 and SPSS for Windows, version 21.0 (SPSS; Chicago, IL, USA).

\section{RESULTS}

Baseline characteristics of the 208 GT3 patients included are summarized in Table 1. Overall, 82\% were male, mean age was 55 years and $98(47 \%)$ had been previously treated with interferon and RBV. In total, $42(20 \%)$ had decompensated liver disease, $55(27 \%)$ had a MELD score $>10$ points and $93(56 \%)$ had a value $>20 \mathrm{kPa}$ on elastography. As to treatment, $131(63 \%)$ received SOF+DCV $\pm \mathrm{RBV}$ and 77 (37\%) SOF/LDV \pm RBV. Therapy duration was 12 or 24 weeks. A significantly larger number of patients received 24 weeks in the SOF/LDV-treated group than the SOF+DCV group. However, a higher percentage of decompensated patients received the $\mathrm{SOF}+\mathrm{DCV} \pm \mathrm{RBV}$ combination. Moreover, patients treated with DCV tended to have higher MELD scores and lower platelet and albumin levels, although the mean elastography value did not differ between the two groups. The percentage of previously treated patients in the two groups was similar. 
Table 1. Baseline characteristics of patients according to the sofosbuvir-based antiviral regimen

\begin{tabular}{|c|c|c|c|c|}
\hline & Total N=208 (\%) & $\mathrm{SOF}+\mathrm{DCV} \pm \mathrm{RBV} \mathrm{N}=131(\%)$ & $\mathrm{SOF} / \mathrm{LDV} \pm \mathrm{RBV} \mathrm{N}=77(\%)$ & $P$ value \\
\hline Male, n (\%) & $170(82)$ & $110(84)$ & $60(78)$ & .18 \\
\hline Mean (SD) age, y & $55(8)$ & $55(8)$ & $55(8)$ & .49 \\
\hline$\geq 65 y, n(\%)$ & $21(10)$ & $14(11)$ & $7(9)$ & .46 \\
\hline Treatment experience, $\mathrm{n}(\%)$ & $98(47)$ & $58(45)$ & $40(53)$ & .19 \\
\hline \multicolumn{5}{|l|}{ CPT classification, $\mathrm{n}(\%)$} \\
\hline A & $166(80)$ & $98(78)$ & $68(88)$ & \multirow{2}{*}{.013} \\
\hline $\mathrm{B} / \mathrm{C}$ & $36(17) / 6(3)$ & $28(21) / 5(4)$ & $8(11) / 1(1)$ & \\
\hline Clinical decompensation, $\mathrm{n}(\%)$ & $41(20)$ & $34(26)$ & $7(9)$ & \multirow{4}{*}{.003} \\
\hline Ascites, $\mathrm{n}$ events & 38 & 31 & 7 & \\
\hline Variceal haemorrhage, $n$ events & 17 & 13 & 4 & \\
\hline Hepatic encephalopathy, $\mathrm{n}$ events & 12 & 11 & 1 & \\
\hline Mean (SD) platelets, per $10 \mathrm{E} 9 / \mathrm{mL}$ & $121(64)$ & $115(60)$ & $136(68)$ & .03 \\
\hline Mean (SD) albumin, mg/dL & $4(0.6)$ & $3.9(0.6)$ & $4.1(0.4)$ & .014 \\
\hline Mean (SD) HCV RNA, $\log _{10} \mathrm{IU} / \mathrm{mL}$ & $6(0.9)$ & $6(0.9)$ & $6(0.8)$ & .98 \\
\hline Median (IQR) MELD score & $8(4)$ & $9(5)$ & $7.5(2)$ & $<.001$ \\
\hline MELD > 10, n (\%) & $55(27)$ & $46(36)$ & $9(12)$ & $<.001$ \\
\hline Mean (SD) elastography, $\mathrm{kPa}^{\mathrm{a}}$ & $27(15)$ & $27(15)$ & $26(15)$ & .59 \\
\hline Elastography >20 kPa, n (\%) & $93(56)$ & $57(58)$ & $36(53)$ & .31 \\
\hline \multicolumn{5}{|l|}{ Treatment duration, $\mathrm{n}(\%)$} \\
\hline 12 weeks & $50(24)$ & $37(28)$ & $13(17)$ & \multirow{2}{*}{.044} \\
\hline 24 weeks & $158(76)$ & $94(72)$ & $64(83)$ & \\
\hline Addition of RBV, n (\%) & $178(86)$ & $105(80)$ & $73(95)$ & .002 \\
\hline
\end{tabular}

${ }^{\text {a }}$ Available in 165 patients

CPT, Child-Pugh-Turcotte; DCV, daclatasvir; LDV, ledipasvir; MELD, model for end-stage liver disease; RBV, ribavirin; SOF, sofosbuvir

\subsection{Efficacy analysis}

\subsubsection{Sustained virological response}

The SVR12 rate in the overall cohort was 93.8\% (195/208): 94\% (123/131) in patients treated with $\mathrm{SOF}+\mathrm{DCV} \pm \mathrm{RBV}$ and $93.5 \%(72 / 77)$ in those treated with SOF/LDV \pm RBV. SVR12 rates according to the DAA combination received, treatment duration and RBV addition are shown in Figure 1. In decompensated patients, SVR 12 was $90.5 \%$. In compensated patients, SVR12 was similar for both DCV and LDV (94\% vs 96\%, $P=.45$ ). SVR12 was somewhat higher in decompensated patients treated with DCV, although the difference did not reach statistical significance ( $94 \%$ vs $78 \%, P=.2$ ). SVR12 rates according to treatment group and CPT classification are summarized in Figure 2. 


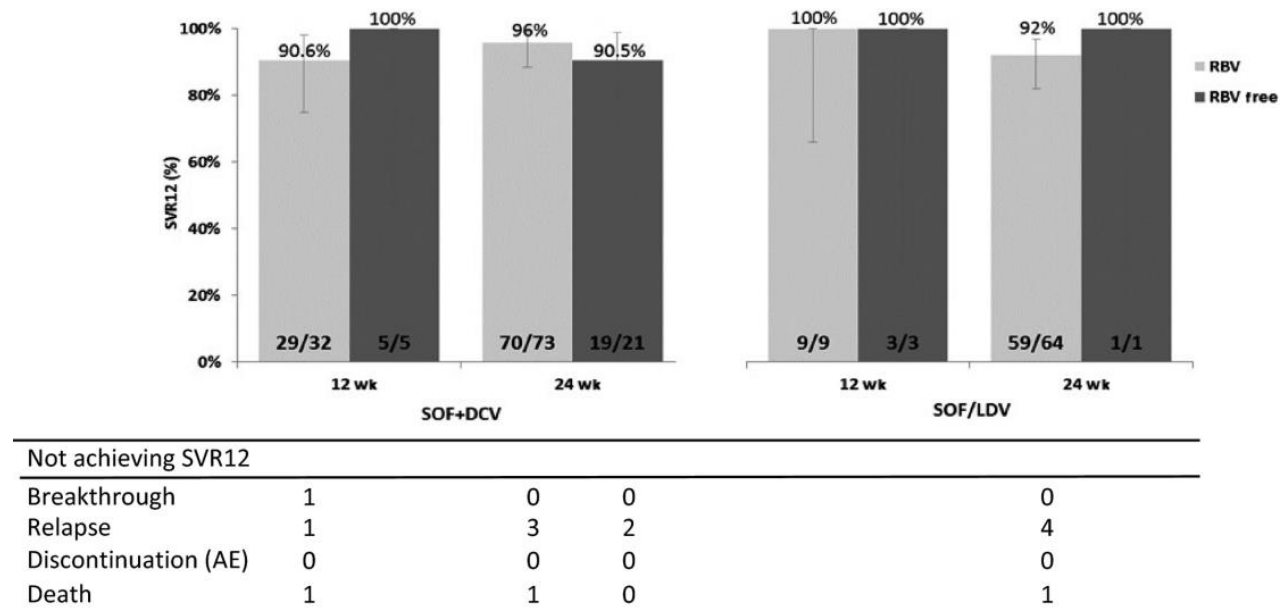

Figure 1. SVR12 rates by intention-to-treat analysis according to NS5A regimen and duration of therapy

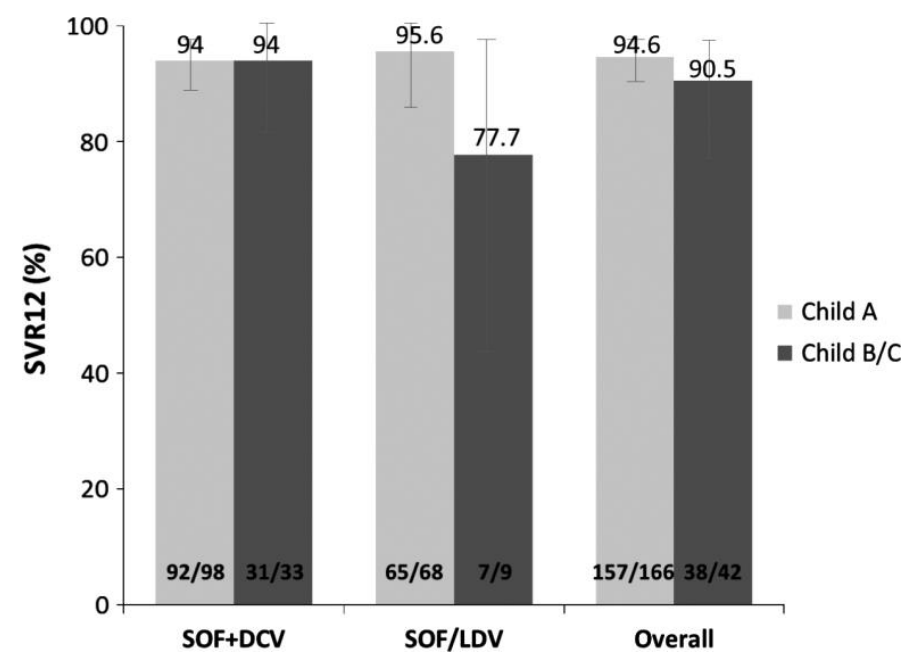

Figure 2. SVR12 rates by intention-to-treat analysis according to NS5A inhibitor regimen and Child-Pugh-Turcotte score

Eleven patients experienced treatment failure, including 10 relapses and one virological breakthrough. In the SOF+DCV \pm RBV group, six patients relapsed and one had detectable HCV RNA at the end of treatment. This last patient was a 56-year-old woman with poor adherence to therapy. Five of the six relapses occurred in patients who received a 24 -week regimen. Of the 77 patients who received $\mathrm{SOF} / \mathrm{LDV} \pm \mathrm{RBV}, 4(5.2 \%)$ relapsed, all after a 24-week regimen with addition of RBV.

Treatment-experienced patients achieved high SVR rates with both DCV and LDV: 55/58 (94\%) and $36 / 40(90 \%)$, respectively. 


\subsubsection{Factors associated with SVR12}

On univariate analysis, platelet count below $75 \times 10 \mathrm{E} 9 / \mathrm{mL}$ was the only factor significantly associated with failure to achieve SVR12 (RR: 3.50, 95\% CI 1.23-9.94, $P=.019$ ). Prior treatment, clinically significant portal hypertension (elastography $>20 \mathrm{kPa}$ ), MELD score and albumin level did not have an impact on SVR12. Neither RBV addition nor treatment duration was associated with therapy failure (Table 2).

Table 2. Univariate analysis of factors associated with SVR12. Platelet count above 75x10E9/mL was the only factor significantly associated to SVR 12 at univariate analysis

\begin{tabular}{lll}
\hline & \multicolumn{2}{l}{ Univariate modified Poisson regression } \\
\cline { 2 - 3 } Variable & RR $(95 \% \mathrm{CI})$ & $P$ value \\
& & \\
& & .912 \\
NS5A inhibitor: DCV vs LDV & $1.06(0.36-3.14)$ & .783 \\
Sex: male vs female & $1.23(0.28-5.34)$ & .769 \\
Age, y: $<65$ vs $\geq 65$ & $1.35(0.18-9.9)$ & .666 \\
Previous therapy: naïve vs experienced & $1.26(0.44-3.63)$ & .919 \\
Addition of $\mathrm{RBV}:$ yes vs no & $1.08(0.25-4.64)$ & .933 \\
Therapy duration, weeks: 12 vs 24 & $1.05(0.3-3.69)$ & .329 \\
Cirrhosis: compensated vs decompensated & $1.76(0.57-5.44)$ & .102 \\
MELD score: $<10$ vs $\geq 10$ & $2.40(0.84-6.83)$ & .342 \\
Elastography, $\mathrm{kPa}:<20$ vs $\geq 20$ & $1.90(0.51-7.11)$ & .019 \\
Platelet count, $\times 10 \mathrm{E} 9 / \mathrm{mL}:<75$ vs $\geq 75$ & $\mathbf{3 . 5 0}(\mathbf{1 . 2 3 - 9 . 9 4})$ & .366 \\
Albumin level, $\mathrm{mg} / \mathrm{dL}:<3.5$ vs $\geq 3.5$ & $1.68(0.54-5.22)$ & \\
\end{tabular}

DCV, daclatasvir; LDV, ledipasvir; MELD, model for end-stage liver disease; RBV ribavirin.

\subsubsection{Subgroup analysis}

To avoid interaction effects resulting from higher percentages of patients with MELD $>10$, platelets $<75 \times 10 \mathrm{E} / \mathrm{mL}$, CPT B/C status and albumin $<3.5$ in the group treated with $\mathrm{SOF}+\mathrm{DCV} \pm \mathrm{RBV}$, we carried out subgroup analyses and multivariate models. A significant interaction was observed between the type of NS5A and MELD score. In the models adjusted by interaction and confounding variables, including platelet count and type of treatment, we found that in patients with a MELD score $<10$, the type of NS5A inhibitor did not have an impact on SVR12 (97\% vs 94\%; adjusted SVR RR 0.49, 95\% CI 0.10-2.45; adjusted risk difference 3.1\%). However, in patients with a MELD score $>10$, the SVR12 rate was lower in those who received SOF/LDV (66.7\% vs 93.5\%; adjusted no-SVR SOF/LDV vs SOF+DCV RR 5.00, 95\% CI 1.19-21.00; adjusted risk difference 26.7\%) (Table 3). 
Table 3. Analysis of SVR12 adjusted to effect-modifying variables

\begin{tabular}{|c|c|c|c|c|c|}
\hline & & $\begin{array}{l}\text { SOF+DCV } \\
\mathrm{N}=123(\%)\end{array}$ & $\begin{array}{l}\text { SOF/LDV } \\
\mathrm{N}=72(\%)\end{array}$ & $\begin{array}{c}P \text { value } \\
\text { Interaction term }\end{array}$ & $\begin{array}{c}\text { Adjusted no-SVR RR } \\
(95 \% \mathrm{CI})\end{array}$ \\
\hline \multirow[t]{2}{*}{ MELD score $^{\mathrm{a}}$} & $\leq 10$ & $77(94)$ & $66(97)$ & .035 & $0.5(0.1-2.5)$ \\
\hline & $>10$ & $43(94)$ & $6(67)$ & & $5.0(1.2-21.0)$ \\
\hline \multirow[t]{2}{*}{ Platelets, $10 \mathrm{E} 9 / \mathrm{mL}^{\mathrm{b}}$} & $\geq 75$ & $88(96)$ & $53(96)$ & .250 & $0.8(0.2-4.4)$ \\
\hline & $<75$ & $35(90)$ & $7(70)$ & & $2.9(0.8-11.1)$ \\
\hline \multirow[t]{2}{*}{ CPT score } & A & $92(94)$ & $65(96)$ & .160 & $0.7(0.2-2.8)$ \\
\hline & $\mathrm{B} / \mathrm{C}$ & $31(94)$ & $7(78)$ & & $3.7(0.6-22.6)$ \\
\hline \multirow[t]{2}{*}{ Albumin, mg/dL } & $>3.5$ & 87 (94) & $67(96)$ & .074 & $0.7(0.2-2.6)$ \\
\hline & $\leq 3.5$ & $34(94)$ & $5(71)$ & & $5.1(0.9-30.8)$ \\
\hline
\end{tabular}

Data available in $205^{\mathrm{a}}$ and $196^{\mathrm{b}}$ patients.

CPT, Child-Pugh-Turcotte; DCV, daclatasvir; LDV, ledipasvir; MELD, model for end-stage liver disease; SOF, sofosbuvir.

\subsection{Safety analysis}

Thirteen (6.3\%) patients experienced serious adverse events (SAEs), including three deaths (1.4\%). Two deaths occurred during the course of antiviral therapy, one in a decompensated patient treated with SOF+DCV who died due to sepsis and another in a decompensated patient who received SOF+LDV and died at home from unknown causes. The remaining death, which occurred in the post-transplantation period, was a CPT A patient with chronic HCV infection who had relapsed previously. The difference in overall SAE rates between patients receiving DCV or LDV did not reach statistical significance $(7.6 \%$ vs $3.9 \% ; P=.38)$. Only one $(0.5 \%)$ patient discontinued therapy because of SAEs, although SVR was achieved. SAEs and deaths were both higher in decompensated patients $(16.7 \%$ vs $3.6 \%, P=.006$ and $4.8 \%$ vs $0.6 \%, P=.04$, respectively). RBV dose reductions in response to haemoglobin decreases were not recorded; nonetheless, only nine of $178(5 \%)$ patients who received RBV had SAEs, compared to four of 30 patients $(13 \%)$ who were not given RBV. The main side effects are shown in Table 4.

Table 4. Serious adverse events (SAE) recorded throughout the period of treatment and 12-week follow-up

\begin{tabular}{lccc}
\hline & Total N=208 (\%) & Child A N=131 (\%) & Child B/C N=77 (\%) \\
\hline SAE, n patients (\%) & $13(6.3)$ & $6(3.6)$ & $7(16.7)$ \\
Hepatic decompensations, n (\%) & $7(3.4)$ & $3(2.3)$ & $4(5.2)$ \\
Infections, n (\%) & $2(1)$ & $1(0.7)$ & $1(1.3)$ \\
Liver-unrelated SAE, n (\%) & $1(0.5)$ & $1(0.6)$ & $0(0)$ \\
Deaths, n (\%) & $3(1.4)$ & $1(0.6)$ & $2(4.7)$ \\
Drug discontinuations, n (\%) & $1(0.5)$ & $1(0.6)$ & $0(0)$ \\
Liver transplantation, n (\%) & $1(0.5)$ & $1(0.6)$ & $0(0)$ \\
Hepatocellular carcinoma, n (\%) & $4(2.6)$ & $2(1.7)$ & $2(5.6)$ \\
\end{tabular}


Fourteen (7\%) patients had a history of HCC prior to the start of antiviral therapy. Although the study was not conducted to assess HCC progression after antiviral therapy, none of these patients had a documented HCC recurrence. In addition, four (2.6\%) patients were diagnosed with HCC during the study period. With the exception of the patient who died in the post-transplantation period, none of the remaining patients had received a liver transplant at the end of the study period.

\section{DISCUSSION}

Patients with HCV GT3 infection and liver cirrhosis are a challenge to treat, particularly those who are treatment experienced. Up to now, there have been few available regimens for GT3 patients. International guidelines now recommend $\mathrm{SOF}+\mathrm{DCV}$ and $\mathrm{RBV}$ for 12-24 weeks for these patients, or SOF/RBV for 24 weeks, or even an interferon-based regimen in combination with RBV and SOF. ${ }^{16,17}$ The advantage of the present study is that it is one of the few that have evaluated 24 weeks of SOF/LDV and RBV in GT3 patients with liver cirrhosis in the real-world setting. The study showed excellent SVR rates in this population when the SOF/LDV plus RBV regimen was given for 24 weeks, with values similar to those achieved with SOF+DCV, particularly in compensated patients. Both regimens were well tolerated, with low associated SAE rates, including deaths and therapy discontinuation.

These results contrast with those obtained in previous studies, which have shown lower SVR rates in GT3 cirrhotic patients treated with SOF+DCV, and particularly with SOF/LDV. It is possible that the shorter treatment duration and absence of RBV in previous studies could explain SVR12 rates of $63 \%$ and $77 \%$ in patients treated with DCV- and LDV-containing regimens, respectively. ${ }^{8}{ }^{10}$ However, neither RBV addition nor 16 weeks duration in patients with advanced liver disease treated in the ALLY-3+ $\operatorname{trial}^{9}$ achieved such high SVR rates.

It is important to note that $20 \%$ of patients from our cohort had decompensated liver disease. In the French cohort, ${ }^{11}$ extended duration of SOF+DCV treatment to 24 weeks in 222 cirrhotic patients achieved an SVR12 rate of $85 \%$ vs $73 \%$ for the 12 -week regimen, with no apparent benefit from adding RBV to the regimen. These rates seem noticeably suboptimal considering the lower percentage of decompensated patients in comparison with our cohort. However, some notable differences between the cohorts may explain these divergent results: in the French study, data regarding the CPT category were missing in $17.8 \%$ of patients, and $15.8 \%$ of the cohort had HIV-HCV coinfection. The findings in 102 patients enrolled in the European compassionate programme showed similar SVR12 results-87\% with SOF+DCV for 24 weeks with or without RBV - and likewise, more than $50 \%$ of patients with cirrhosis were classified as CPT B or C and $15 \%$ had HIV-HCV coinfection. ${ }^{18}$ We observed no differences in SVR12 between patients treated for 12 or 24 weeks or between those receiving or not RBV when DCV was the NS5 inhibitor used.

Although SVR12 was $100 \%$ in patients treated with SOF/LDV with or without RBV for 12 weeks, the limited number of patients included does not allow conclusions to be drawn. However, the SVR12 rate after 24 weeks of SOF/LDV plus RBV was $92.2 \%$, similar to that of SOF+DCV regimens. In contrast to DCV, LDV has shown relatively low in vitro activity against HCV GT3, and it may be that both RBV addition and extension to 24 weeks are needed to achieve comparable SVR12 rates.

Because of the differing baseline characteristics between patients receiving the two regimens, we are unable to carry out direct comparisons; a higher percentage of patients in the group treated with DCV had decompensated cirrhosis, MELD score $>10$ and albumin concentration $<3.5 \mathrm{~g} / \mathrm{dL}$. The explanation for this important limitation of our study may be that the SOF+DCV compassionate programme allowed patients with more advanced liver disease to receive all oral therapies before SOF/LDV had been approved. Nevertheless, subgroup analysis and the adjusted model in patients with a MELD score <10 showed no impact of the type of NS5A inhibitor. The risk of virological failure was higher in patients with MELD >10 receiving LDV. However, because of the limited number of patients who received SOF/LDV, we were unable to determine whether the effectiveness of DCV regimens was higher in decompensated patients. On analysis of several covariates, the only factor associated with a lower 
likelihood of achieving SVR12 was platelet count below $75 \times 10 \mathrm{E} 9 / \mathrm{mL}$. Of note, the low virological failure rates associated with DAAs make it difficult to encounter statistical differences.

In our cohort, $20 \%$ of patients were decompensated, and even in this group, the overall SVR12 rate remained high $(90.5 \%)$. This value is much higher than the SVR12 rates of $70 \%$ and $33 \%$ obtained in decompensated patients treated for 24 or 12 weeks, respectively, in the French cohort. ${ }^{11}$ It is also higher than the $71 \%$ and $59 \%$ SVR12 values reported in the British cohort treated with a DCV or LDV combination, respectively, for 12 weeks. ${ }^{12}$ Although the shorter treatment duration in the British cohort may explain the results in LDV patients, the SVR12 rates in our patients treated with SOF+DCV for 12 weeks were also higher. Poorer liver function in the British cohort would not explain the differences, as the median MELD score, and albumin and platelet values were similar to those in our cohort.

Despite a longer therapy duration and addition of RBV in most of our patients, the SAE rate was remarkably low. There were only two deaths while on treatment, one of them likely unrelated to drug toxicity. Of the 13 patients with SAEs, 11 completed the scheduled treatment. The nonsignificantly higher SAE rate observed in the DCV group was probably linked to the higher percentage of decompensated patients treated with this combination. CPT B or C patients were more likely to present SAEs (17\%), most of them as clinical decompensation. In the British cohort, which included only CPT B or C patients, the SAE rate was $25 \%$.

Recent studies using the new NS5A inhibitor, velpatasvir (VEL), in combination with SOF have reported SVR12 rates similar to those obtained in the present cohort: SVR12 was 89\% in treatmentexperienced cirrhotic patients treated for 12 weeks ${ }^{19}$ and $85 \%$ in CPT B decompensated patients treated with SOF/VEL plus RBV for 12 weeks. ${ }^{20}$ In the latter study, the 12- and 24-week regimens without RBV achieved a very low SVR12 rate (50\%), although only 39 GT3 patients in total were included.

In conclusion, in this real-life cohort study, both SOF+DCV and SOF/LDV with RBV during 24 weeks were safe and highly effective treatments for HCV GT3 patients with compensated cirrhosis. Based on these findings, it seems that both these regimens can be safely recommended for this subset of patients.

\section{ACKNOWLEDGEMENTS}

Writing support was provided by Fidelma Greaves and Celine Carvalho. Statistical analysis support was provided by Elia Pérez Fernández.

\section{REFERENCES}

1. Bochud PY, Cai T, Overbeck K, et al. Genotype 3 is associated with accelerated fibrosis progression in chronic hepatitis C. J Hepatol. 2009;51:655-666.

2. Nkontchou G, Ziol M, Aout M, et al. HCV genotype 3 is associated with a higher hepatocellular carcinoma incidence in patients with ongoing viral C cirrhosis. J Viral Hepat. 2011;18:e516e522.

3. Probst A, Dang T, Bochud M, Egger M, Negro F, Bochud PY. Role of hepatitis C virus genotype 3 in liver fibrosis progression-a systematic review and meta-analysis. J Viral Hepat. 2011;18:745-759.

4. Sofia MJ, Bao D, Chang W, et al. Discovery of a beta-d-2'-deoxy-2'-alpha-fluoro-2'-beta-Cmethyluridine nucleotide prodrug (PSI-7977) for the treatment of hepatitis C virus. J Med Chem. 2010;53:7202-7218.

5. Gao M, Nettles RE, Belema M, et al. Chemical genetics strategy identifies an HCV NS5A inhibitor with a potent clinical effect. Nature. 2010;465:96-100.

6. Harvoni (ledipasvir and sofosbuvir) [summary of product characteristics]. Cambridge, UK: Gilead Sciences International Ltd, 2014. 
7. Hernandez D, Zhou N, Ueland J, Monikowski A, McPhee F. Natural prevalence of NS5A polymorphisms in subjects infected with hepatitis $\mathrm{C}$ virus genotype 3 and their effects on the antiviral activity of NS5A inhibitors. J Clin Virol. 2013;57:13-18.

8. Nelson DR, Cooper JN, Lalezari JP, et al. All-oral 12-week treatment with daclatasvir plus sofosbuvir in patients with hepatitis C virus genotype 3 infection: ALLY-3 phase III study. Hepatology. 2015;61:1127-1135.

9. Leroy V, Angus P, Bronowicki J, et al. Daclatasvir, Sofosbuvir, and Ribavirin for hepatitis C virus genotype 3 and advanced liver disease: a randomized phase III study (ALLY-3+). Hepatology 2016;63:1430-1441.

10. Gane EJ, Hyland RH, An D, et al. Efficacy of ledipasvir and sofosbuvir, with or without ribavirin, for 12 weeks in patients with HCV genotype 3 or 6 infection. Gastroenterology. 2015;149:1454-1461 e1.

11. Hezode C, De Ledinghen V, Fontaine E, et al. Dacltasvir plus Sofosbuvir with or without ribavirin in patients with $\mathrm{HCV}$ genotype 3 infection: interim analysis of a French multicenter compassionate use program. Hepatology. 2015;62:314A.

12. Foster GR, McLauchlan J, Irving W, et al. Treatment od decompensated HCV cirrhosis in patients with diverse genotypes: 12 weeks sofosbuvir and NS5A inhibitors with/without ribavirin is effective in HCV genotypes 1 and 3. J Hepatol. 2015;62:S190.

13. Bureau C, Metivier S, Peron JM, et al. Transient elastography accurately predicts presence of significant portal hypertension in patients with chronic liver disease. Aliment Pharmacol Ther. 2008;27:1261-1268.

14. Lemoine M, Katsahian S, Ziol M, et al. Liver stiffness measurement as a predictive tool of clinically significant portal hypertension in patients with compensated hepatitis $\mathrm{C}$ virus or alcohol-related cirrhosis. Aliment Pharmacol Ther. 2008;28:1102-1110.

15. Zou G. A modified Poisson regression approach to prospective studies with binary data. Am J Epidemiol. 2004;159:702-706.

16. AASLD/IDSA/IAS-USA guidelines. 2016; www.hcvguidelines.org. Accessed 24 Feb, 2016.

17. European Association for Study of Liver. EASL recommendations on treatment of hepatitis C 2015. J Hepatol. 2015;63:199-236.

18. Welzel TM, Herzer K, Ferenci P, et al. Daclatasvir plus sofosbuvir with or without ribavirin for the treatment of $\mathrm{HCV}$ in patients with severe liver disease: interim results of a multicenter compassionate use program. J Hepatol. 2015;62:S619.

19. Foster GR, Afdhal N, Roberts SK, et al. Sofosbuvir and velpatasvir for HCV genotype 2 and 3 infection. N Engl J Med. 2015;373:2608-2617.

20. Curry MP, O'Leary JG, Bzowej N, et al. Sofosbuvir and velpatasvir for HCV in patients with decompensated cirrhosis. N Engl J Med. 2015;373:2618-2628. 